PREHOSPITAL CARE

\title{
The emerging role of the emergency care practitioner
}

\author{
S Cooper, B Barrett, S Black, C Evans, C Real, S Williams, B Wright
}

Emerg Med J 2004;21:614-618. doi: 10.1136/emj.2003.011247

See end of article for authors' affiliations .....................

Correspondence to: Dr S Cooper, Faculty of Health and Social Work, C403 Portland Square, University of Plymouth, Plymouth, Devon PL4 8AA, UK; simon.cooper@ plymouth.ac.uk

Accepted for publication 13 April 2004
A mbulance services across the UK are increasingly aware of the need to evaluate their educational provision and to consider new and innovative operational practice. Government ministers have been emphasising the importance of team working, maximising the contribution of all staff to patient care, modernising education and training, and expanding the workforce. ${ }^{1-3}$ Pressure is being applied on all those involved in healthcare education to produce personnel who are "fit for practice" within a seamless service and to break down what Alan Milburn calls "tribalism", ${ }^{45}$ reducing the barriers between professions and specialties.

Issues concerning paramedic education are being raised especially the need for a greater decision making repertoire and "a greater emphasis on deciding whether or not to treat patients before transporting to hospital" .

One of the most influential publications has been the Joint Royal Colleges Ambulance Liaison Committee (JRCALC) report $^{7}$ on the need for an advanced practitioner in emergency care (PEC), (now referred to as an emergency care practitioner (ECP)) encompassing issues related to advanced autonomous practice and multi-professional roles and links, based upon a higher education curriculum. The UK government's Changing Workforce Programme has been actively promoting practitioner developments and foresees a number of advantages within the role including reduced accident and emergency (A\&E) attendances, improved patient care, and increased operational efficiency.

With these issues in mind the University of Plymouth (UK) has been running the first multi-professional BSc in Emergency Care since 2000. Between 8 and 10 students have been recruited to each cohort-50\% A\&E nurses and 50\% paramedics. The two year part time programme is made up of seven modules covering issues such as core concepts in emergency care, mentorship, applied skills in emergency care, and leadership and management. Core competencies for paramedic practitioners have been incorporated into the programme based on the ongoing work of the Higher Education Ambulance Development Group (internet appendix 1, http://www.emjonline.com/supplemental). Advanced assessment and patient management skills are a key focus, for example, triage/prioritisation techniques, wound closure, health promotion, utilisation of support services, and major incident management.

Despite these innovations the role of the ECP remains unclear. This paper reports on an evaluation of the role within Westcountry Ambulance Service NHS Trust (UK).

\section{METHODS}

On graduation four experienced (mean 10 years/range 9-12) male paramedics were appointed to the practitioner role in September 2002 with an open remit to explore and develop the role. Two were based in West Cornwall and two in West Devon. The two regions serve a population of about half a million in Cornwall and 2 million in Devon, with the ambulance trust employing 741 front line A\&E staff in the two counties. The Cornish based practitioners practised in predominantly rural populations while the Devon contingent practised in an urban population (Plymouth) and the rural community of East Cornwall. All the practitioners were based in a local minor injury unit (MIU) for half their working week and their base ambulance station for the remainder. The secondment to the MIUs was in response to local primary care trusts interest and support for the role and as an additional development opportunity for the ECPs. Methods of

Abbreviations: ECP, emergency care practitioner; EMS, emergency medical system; MIU, minor injury unit; PTS, patient transport system; RRV, rapid response vehicle; RITA, rapid intervention, treatment, and assessment; UTV, urgent transport vehicle 
transportation varied; motorbike, rapid response vehicle (RRV), air ambulance, or a practitioner car (a converted Volvo estate for the treatment of patients on scene).

Data collection ran for six months (October 2002 to March 2003 ) and incorporated a period of operational development and refinement before the activation of the patient care phase. Using both qualitative and quantitative approaches the objective was to undertake a scoping exercise to identify the scope of practice of the ECP and to compare that with the paramedic, and to begin to understand the possible impact of ECPs on the patient's emergency care experience. Clearly the roles of ECP and paramedic will differ, the question was how did they differ and was their a benefit?

The programme was designed to evaluate a developing situation, in which the views and experiences of the people involved would be an essential part of the data. To this end a constructivist methodology was chosen. ${ }^{89}$ This approach takes stakeholder inputs (claims, concerns, and issues) as organisational and developmental foci for the evaluation, drawing upon the constant comparisons of different groups constructions of realities and thereby capturing the dynamics of rapidly changing circumstances and environments.

Two stages of data collection were developed, the first was based upon the four practitioners' reflective reports, MIU documentation, and adapted patient report forms (PRFs). PRFs from this first case group were compared with those completed by 11 paramedics (five from the East and six from the West). Based on a criterion sample of experienced $(>3$ years) paramedics, 12 staff were initially recruited with 11 completing the relevant documentation; their experience ranged from 3-15 years (mean 8 years) which was comparable to that of the ECPs. The second phase of data collection was based upon individual and focus groups interviews with key stakeholders, ECPs, paramedics, ECP managers, MIU and A\&E staff.

Data were analysed using the constant comparison method, ${ }^{9}$ filed notes and transcripts were coded, and concepts compared with the aid of QSR N5 (Scolari, London). Emerging concepts influenced the data collection and sampling as the process developed. The objective was to produce analytical generalisation ${ }^{10}$ rather than statistical generalisability. However, statistical information was gained from the practitioner and paramedic adapted PRFs and was analysed with the use of the SPSS. Univariate analysis of nominal data was performed using $\chi^{2}$ analysis with Yates's correction, ordinal data with Spearman (p) rank correlation. These two elements of data were compared ensuring that the qualitative issues were explained in relation to the statistical evidence.

\section{RESULTS}

Four ECPs and 11 paramedics participated in the data collection. For incidents within the emergency medical system (EMS) control, an adapted PRF was completed. Data were taken from the MIU patient record for the practitioners' MIU cases.

The paramedics reported on 331 cases while the ECPs reported on 170 cases within the EMS system and 191 MIU cases.

\section{Patient demographics}

Of the 692 cases, $51 \%$ of patients were male and $44 \%$ female. Patient age ranged from $<1$ year to 99 years. ECPs and paramedics exposure to adult patients did not differ. However, ECPs did have more exposure to patients under the age of 16 years $(p=0.001)$ mainly attributed to their MIU work.

\section{Chief complaints}

Table 1 shows the range of conditions seen from the EMS. ECPs saw significantly less "other medical" patients than paramedics $(p=0.017)$ and significantly more trauma cases $(\mathrm{p}=0.014)$. Internet appendix 2 (http//www.emjonline.com/ supplemental) shows the range of conditions seen by the ECPs in MIU of which 30\% (57 of 191) of patients had lacerations, and 35\% (67 of 191) presented with soft tissue or ligament injuries.

\section{Deployment}

There were differences between the way in which the ECPs and paramedics were deployed $(\mathrm{p}=0.000)$. Paramedics were resourced by ambulance control, while ECPs, for example, were self activated $45 \%$ (76 of 170) of the time and by ambulance control 34\% (58 of 170) of the time. Attending crews (including those in rapid response vehicles (RRVs)) called for ECP assistance in 18\% (31 of 170) of cases.

\section{Discharge}

ECPs were more likely to treat patients on scene than paramedics $(p=0.007) ; 28 \%$ (48 of 170$)$ by ECPs compared with $18 \%$ (59 of 331) by paramedics. None of the ECPs' or paramedics' patients were subsequently conveyed within 24 hours. However, there was a repeat call to an ECP's patient who had fallen for a second time.

Patients were conveyed, either by A\&E, urgent transfer, patient transfer, rapid response, air ambulance, or ECP vehicles. ECPs arranged conveyance for $50 \%$ (85 of 170) of their patients while paramedics conveyed $64 \%$ (212 of 331) $(\mathrm{p}=0.000)$. This paramedic comparison group conveyance rate was comparable to the Trust wide conveyance rate, which for the period of the study equated to $67 \%$. Table 2 breaks down the recorded discharge data including the referral and conveyance rates.

ECPs submitted a total of 269 reflective case reports. Individual and focus groups interviews were performed with the four ECPs, four ECP managers, one senior member of the MIU staff, one A\&E consultant, and four paramedics who were not part of the comparison group. (The following quotations are coded as either "ECP reflective" (ECPr) or "Stakeholder" (St), which may be manager (Man) or clinician (Clin).

Four key themes emerged from the data;

- Resource deployment

- Transportation

- Communication

Table 1 Chief complaints (EMS incidents)

\begin{tabular}{lll}
\hline Chief complaint & $\begin{array}{l}\text { Number of patients } \\
\text { seen by paramedics }\end{array}$ & $\begin{array}{l}\text { Number of patients } \\
\text { seen by ECPs }\end{array}$ \\
\hline Respiratory & 34 & 14 \\
Cardiac & 45 & 21 \\
Gastrointestinal & 14 & 4 \\
Neurological & 24 & 6 \\
Other medical & 55 & 15 \\
Trauma & 72 & 54 \\
Burns & 4 & 2 \\
Poisoning & 3 & 1 \\
Deliberate self harm & 9 & 2 \\
Social need & 11 & 7 \\
Other unspecified & 12 & 2 \\
condition & & \\
No information & 48 & 42 \\
Total & 331 & 170 \\
\hline
\end{tabular}




\begin{tabular}{|ll|}
\hline Table 2 ECPs patient discharge records & \\
\hline Treated on scene & 48 \\
Referred to general practitioner & 10 \\
Referred to police & 1 \\
Referred to bereavement team & 1 \\
Referred to rapid intervention treatment & 4 \\
and assessment (RITA) team (RITA) & 5 \\
Non-iniury/no treatment & 6 \\
Patients downgraded for a later response & 6 \\
Deceased & 2 \\
Children transported to A\&E/MIU by parents & 85 \\
Conveyed to A\&E/MIU by ambulance service & 2 \\
No discharge data & 170 \\
Total & \\
\hline
\end{tabular}

- Training and education

- Inter-agency cooperation collaboration

- Patient care

\section{Resource deployment \\ Transportation}

Practitioners were deployed by various means; either by direct orders from ambulance control, self activation from pager information, or by request from ambulance crews through mobile phones. A major reported benefit of the ECP role was the reduction in unnecessary trips by emergency ambulance to A\&E and an ability to re-grade incidents appropriately.

The training, competence, and confidence of the ECPs appeared to improve their decision making repertoire with a significant impact on resources. The following quote illustrates these issues:

\begin{abstract}
"Great to do some wound care in the field. Released the crew - they were happy. With the family we discussed a plan. I then liaised with Kernodoc over the phone who were in agreement with me and facilitated a bed in the local community hospital. I took the fairly mobile lady in my practitioner car to the hospital, admitted her and handed her over to the staff. Had freed up WAST (Westcountry Ambulance Service Trust) resource to do other work. This crew would have undoubtedly taken her to a district general hospital A\&E which would have been inappropriate for a 96 year old lady" (ECPr).
\end{abstract}

A notable event was the redeployment and local management of resources on New Year's Eve 2002. The "999" calls to WAST from the town of St Ives in West Cornwall were directed to the on-scene practitioner's mobile phone. This enabled the practitioner to allocate resources appropriately on a local level. Twenty patients were seen of which only five were transported to A\&E using four vehicles.

Practitioners were also active in "standing down" emergency vehicles on "red" 999 calls, reducing the risk to the public. They also cited the benefits of giving patients time to recover instead of rushing them into an ambulance and the local A\&E department. Benefits were also perceived from the allocation of patient transport system (PTS) vehicles and staff to the ECPs reducing the impact on A\&E transport.

\section{Resource deployment}

\section{Communication}

In the early days of the trial concerns were raised over the allocation of resources and methods of deployment. Ambulance control had ECPs placed on the "back screen" classified as "other" resources, leading to long periods of inactivity for the practitioners.

"Self activated via my page. Didn't bother to ring control as they are not proactive and it takes too long. Control never phoned me once" (ECPr).

Adjusting to accommodate the emerging role of the practitioner was a challenge, not helped by the scarcity of practitioners, irregular availability, and the uncertain nature of their role. This was partially resolved by the introduction of a "response bureau" based in control, where experienced officers were appointed to identify suitable calls for the ECPs. However, practitioners argued the need for flexibility and the freedom to make their own dispatch decisions based on local knowledge.

\section{Training and education}

ECPs and stakeholders agreed that the additional graduate level training the ECPs had received improved their clinical practice. There were also reported benefits from the experienced gained working in MIUs;

"... prior to the degree course, I would not have been able to treat a patient with the confidence and competence required. However, having studied both anatomy physiology and clinical evaluation I was in a strong position to treat this patient appropriately" (ECPr).

"The wound did look big but I have treated bigger wounds in the MIU so I knew exactly what to do. I think I may have been fazed had I not have had that MIU experience" (ECPr).

The ECPs appeared to have developed skills in leadership and reported a level of influence over their own colleagues and in the multi-professional arena, for example;

"The degree and appointment as a practitioner has empowered me-lt gives me the authority and confidence to take decisions" (ECPr).

ECPs also appeared to be considering their practice based upon the latest evidence;

"...an experienced paramedic began IV placement before leaving the scene. The current EMJ (Emergency Medicine Journal) has an article referring to the best evidence for fluid therapy ...There was no real need for fluid in this patient and I was really wondering whose benefit he was cannulating for" (ECPr).

Practitioner reports were reflective in nature and often identified areas of weakness, for example, wound care, ionising and radiation issues, radiological interpretation, some advanced examination techniques and suturing skills. Over time these issues were resolved. However, prescribing issues especially in relation to antibiotics and tetanus immunisation were not resolved.

\section{Inter-agency cooperation and collaboration}

The practitioners were proactive in building inter-agency links and developed patient referral processes with GPs and the local rapid intervention treatment and assessment (RITA) teams (a group made up of social services, occupational therapists, and physiotherapists) with direct admission policies to community hospitals and care facilities. In 
addition they forged links with the community psychiatric service, district nurses, nurse practitioners, physiotherapists, the reablement team, and the falls group (an information based group for falls prevention and awareness). There was evidence to suggest that ECPs were able to identify and initiate actions on behalf of, or in cooperation with, patients and relatives, which might not be identified in current practice between the responsible agencies.

The MIU links were also cited as beneficial to both parties;

\section{"I thought it was a good benefit having them, it was a good learning curve for us plus they were lucky to come down to clinics and get some suturing experience" (St/ Clin)}

Cultural changes relating to emerging roles were an issue, with tensions over the training of practitioners in some UK regions, "on the cheap" (St/Clin), and concerns over the placement of paramedics in traditional nursing and general practice roles. Practitioners reported a cautious approach:

\section{"Once again I was conscious of the need to be circumspect when dealing with the crew that were "first on scene" (ECPr).}

\section{Patient care}

Practitioners and stakeholders reported a variety of benefits to patient care particularly relating to issues around referral processes and treat and release. They organised direct admission to wards and assessment by appropriate agencies (for example, the falls team). Wound care, including glue and steri-stripping, and advanced examination and assessment skills were frequently reported.

No serious errors in patient care were apparent, however, one diagnostic error was mentioned;

\section{"...the bad side was that my diagnosis of a sprain was incorrect and the $\mathrm{x}$-ray showed fracture of the lower fibula" (ECPr).}

In conclusion, one stakeholder summarised the role as follows;

\section{"...I've been in the service 22 years, the only time I can remember anything moving the service forward as much as this was when paramedics first came. The potential for the practitioners is fantastic and for the first time ever there is an opportunity for a real link into the rest of the health system... and being taken seriously by the NHS. If we've got the right people driving forward the practitioner role we will have a massive impact on patient care and delivering quality patient care, and I just think it's a really exciting time to be a part of this new way of thinking and working" (St/Man)}

\section{DISCUSSION}

This study was set at a time when WAST were receiving an average of 13000 calls a month, so clearly our sample is small. We were also studying a small and highly motivated group of people, which may not be the case in the future. However, the results of this study indicate that the ECP role is developing along the lines envisaged by a number of advisory and governing bodies. ${ }^{2367}$ The initial implementation of the role was a significant challenge to the service but ECPs were proactive in their development and made significant efforts to heighten awareness and to resolve some of the tensions inherent in a new role. They were particularly active in their development of inter-agency links and worked closely with ambulance control to resolve communication problems and to identify cases that matched their skills.

There are indications that the role would be best managed by a flexible approach to deployment, and an empowerment of practitioners at a local level. The above report of New Year's Eve 2002 is a good example. Woods ${ }^{11}$ supports this view reporting that the implementation of advanced practice is best facilitated with supernumerary status and the autonomy to organise and undertake practice.

The reader should be cautious in the interpretation of the quantitative comparisons between ECP and paramedic as our primary objective was to analyse the overall emerging role of the ECP. The process of patient referral clearly differed to that of the paramedic and therefore patient cases differed (table 1). It is quite possible, for example, that they were more likely to be attend patients who would be less likely to be conveyed. Of course the negative corollary could have been that despite the investment in ECPs there was no difference in the conveyance rates. However, ECPs did seem to have an impact on the operational activity within the service, particularly relating to referral/release practice and nonconveyance issues, which supports the evidence for alternative referral pathways. ${ }^{12}{ }^{13}$ They were active in "standing down" responders and downgrading calls and were able to manage patient care for the early release of crews. They were more likely to treat patients on scene than paramedics $28 \%$ compared with $18 \%(\mathrm{p}=0.007)$ and were therefore less likely to convey patients, $50 \%$ compared with $64 \%(p=0.000)$. ECP and paramedic figures that compare well with 1999 English and Welsh conveyance rates of $83 \%{ }^{14}$

The quality of care in ECP (and paramedic) treat and release practice does need to be challenged. Other than a second call to a patient who frequently fell, our investigations show that there were no secondary emergency calls (within 24 hours) to practitioners' and paramedics' patients. However, a review of the literature relating to emergency ambulance crews (not including practitioners) by Snooks et $a l,{ }^{15}$ produces evidence that a significant minority of patients who are not conveyed are at risk of deterioration.

As in comparable studies, ${ }^{16}$ graduate level education was cited as applicable and appropriate. Reports indicated that the programme had empowered people and increased competence and confidence. Practitioners took up the mantle of leadership and were active in the education of staff and patients. MIU secondments were reported as an essential component of development, maintaining and improving practitioners' position on the skills escalator. Unfortunately the future of such courses remains uncertain. Ambulance services continue to fund their own pre-qualifying training and educational provision, with pockets of investment from the Workforce Development Confederations and the Higher Education Funding Council for England. As the Ambulance Service Association reported in $2001^{6}$ "training for ambulance paramedics and Practitioners in Emergency Care should be funded through the Non-Medical Education and Training budget, as happens for other Allied Health Professions".

Flexible deployment, reduced inappropriate conveyance, on scene treatment of minor injuries, inter-agency referral mechanisms, and alternative care pathways are likely to produce a more rounded holistic system of care-appropriate care, at an appropriate time in an appropriate place. The results from this study show that an investment in the role could be beneficial. However, more work is required to evaluate the development of practice, the quality of care, and the cost benefits of such schemes, especially those relating to 
educational provision, and for example, extended on scene times in comparison with conveyance costs.

\section{ACKNOWLEDGEMENTS}

Thanks to the 11 WAST paramedics who assisted with data collection, Professor Julie Scholes, University of Brighton, for her guidance and feedback, and to the Changing Workforce Programme for their financial investment in this project.

\section{CONTRIBUTORS}

Simon Cooper initiated the research, discussed core ideas, participated in the protocol design, and coordinated and assisted in the data collection. He assisted in the analysis and interpretation of the data and wrote and edited the paper. Chris Evans and Sarah Black discussed core ideas, participated in the protocol design, assisted in the data collection, undertook the data entry, assisted in the data analysis, and edited the paper. Barry Barrett, Carlton Real, Simon Williams, and Bryan Wright discussed core ideas, assisted in the data collection, and edited the paper. The guarantor of this paper is Simon Cooper.

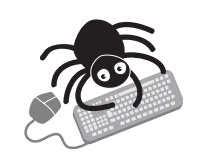

The appendices are available to view on the journal web site (http://www.emjonline.com/supplemental).

\footnotetext{
Authors' affiliations Westcountry Ambulance Service NHS Trust, UK

Funding: the Changing Workforce Programme.

Conflicts of interest: none declared.
}

S Cooper, B Barrett, S Black, C Evans, C Real, S Williams, B Wright,

\section{REFERENCES}

1 Department of Health. A health service for all the talents: developing the NHS workforce. Consultation document on the review of workforce planning. London: Department of Health, 2000.

2 Department of Health. Reforming emergency care. London: Department of Health, 2000.

3 Department of Health. Life in the fast lane. London: Department of Health, 1997.

4 Sanders C. Moves to get the NHS fighting fit. Times Higher Education Supplement 2001;8 Jun.

5 Bulstrode C, Bell Y, Gray M. Senior house officers: the lost tribes. Br J Hosp Med 1993;50:572-3.

6 Ambulance Service Association and Medical Care Research Unit. The future of the ambulance service in the United Kingdom. London: Ambulance Service Association \& Medical Care Research Unit, 2001.

7 Joint Royal Colleges Ambulance Liaison Committee. The future role and education of paramedic ambulance service personnel (emerging concepts). London: Joint Royal Colleges Ambulance Liaison Committee, 2000.

8 Guba EG, Lincoln YS. Fourth generation evaluation. Newbury Park, CA: Sage, 1989.

9 Glaser B, Strauss A. The discovery of grounded theory strategies for qualitative research. London: Weidenfeld and Nicholson, 1967.

10 Firestone WA. Alternative arguments for generalizing from data as applied to qualitative research. Educational Researcher 1993;22:16-23.

11 Woods LP. Implementing advanced practice: identifying the factors that facilitate and inhibit the process. Journal of Clinical Nursing 1998;7:265-73.

12 Marks PJ, Daniel TD, Afolabi O, et al. Emergency (999) calls to the ambulance service that do not result in the patient being transported to hospital: an epidemiological study. Emerg Med J 2002;19:449-52.

13 Dale J, Higgins J, Williams S, et al. Computer assisted assessment advice for "non-serious" 999 ambulance service callers: the potential impact on ambulance dispatch. Emerg Med J 2003;20:178-83.

14 Department of Health. Statistical BulletinAmbulance Services 1998-9. Bulletin. 1999/16. London: Department of Health, 1999.

15 Snooks H, Kearsley N, Halter $M$, et al. On-scene alternatives for ambulance crews attending patients with primary care needs: a review of the literature (in press).

16 Girot EA. Graduate nurses: critical thinkers or better decision makers? J Adv Nurs 2000;31:288-97. 